\title{
Experimental closed system surgical procedures and intraocular pressure fluctuation ${ }^{1}$
}

\author{
Eduardo Vieira de SouzaI, Nivaldo Vieira de Souza ${ }^{I I}$, Maria de Lourdes Veronese Rodrigues ${ }^{\text {III }}$
}

DOI: http://dx.doi.org/10.1590/S0102-86502014001800005

${ }^{I} \mathrm{PhD}$, Assistant Professor, Division of Surgery, Faculty of Medicine, Federal University of Santa Catarina (UFSC), Florianopolis-SC, Brazil. Conception, design, intellectual and scientific content of the study; acquisition, analysis and interpretation of data; manuscript writing; critical revision. ${ }^{I I} \mathrm{PhD}$, Assistant Professor, Ophthalmology Division, Ribeirao Preto Medical School, University of Sao Paulo (USP), Ribeirao Preto-SP, Brazil. Conception, design, intellectual and scientific content of the study; acquisition, analysis and interpretation of data; manuscript writing, critical revision. IIIPhD, Full Professor, Ophthalmology Division, Ribeirao Preto Medical School, USP, Ribeirao Preto-SP, Brazil. Conception, design, intellectual and scientific content of the study; acquisition, analysis and interpretation of data; manuscript writing, critical revision.

\begin{abstract}
PURPOSE: To determine the behavior of intraocular pressure and its relationship with infusion pressure in vitrectomy, phacofragmentation and phacoemulsification surgeries in rabbits.

METHODS: Intraocular pressure fluctuation was measured in 24 eyes of 12 rabbits submitted to vitrectomy, phacofragmentation and phacoemulsification procedures (eight eyes per group). The procedures were divided according to the force of aspiration of the instrument. Intraocular pressure was monitored with a computerized polygraph by means of a cannula introduced into the vitreous chamber.

RESULTS: Intraocular pressure showed a mean variation from $33 \mathrm{mmHg}$ (maximum) to $6 \mathrm{mmHg}$ (minimum). Vitrectomy showed the greatest difference between weak and strong aspiration. The greatest fluctuations occurred during procedures with strong aspiration, with phacoemulsification showing the widest variation, with maximum peaks almost reaching $50 \mathrm{mmHg}$. The infusion pressure varied less than the intraocular pressure, especially at the lowest pressures.
\end{abstract}

CONCLUSIONS: The fluctuation of intraocular pressure during ocular surgeries was not great. The higher the aspiration pressure, the higher the intraocular pressure. The infusion pressure did not show a good correlation with intraocular pressure.

Key words: Intraocular Pressure. Vitreoretinal Surgery. Cataract. Animal Experimentation. Rabbits. 


\section{Introduction}

The last four decades have been marked by significant scientific and technological advances in various areas of knowledge. In medicine, ophthalmology has actively participated in this progress, with important changes due to the introduction of new equipment for the diagnosis and treatment of ocular diseases. The ophthalmologic surgical act has been profoundly modified by the development of new techniques and technologies. Today, cataract surgery is performed with ultrasound and with the implant of an intraocular lens ${ }^{1}$ and retinal surgery is performed by means of microincisions using sophisticated equipment ${ }^{2}$. These new procedures have permitted surgeons to obtain greater precision and control of the surgical act, minimizing the operative trauma, with a consequent improvement of results ${ }^{3}$.

In these types of surgery, intraocular pressure (IOP) is determined only by the infusion force (height of the infusion flask), the caliber of the cannulas, and the aspiration force. Since they manipulate the internal part of the ocular globe by incisions with little or no area of continuity between the inner and outer medium, these techniques have been called "closed system intraocular surgeries"4. The rapid development and refinement of these surgeries, despite excellent results, has not yet permitted a perfect analysis of all the risk factors associated with the procedures. Thus, we must recognize and minimize the effect of these factors, with further improvement of the surgical results.

Variation in pressure is believed to occur during the course of closed system intraocular surgeries, but there is no information about its behavior. The increase in IOP above systolic arterial pressure is known to damage the retina and other ocular tissues ${ }^{5}$. Even with increases in pressure to lower levels (60 to $80 \mathrm{mmHg}$ ), there seems to be impairment of these structures such as a rupture of the water-blood barrier, which may also occur when the pressure is abruptly reduced to very low levels ${ }^{6}$. Thus, wide variations in IOP seem to be directly related to the postoperative inflammatory process ${ }^{7}$. For this reason, the objective of the present study was to determine the behavior of IOP and its relationship with infusion pressure during the course of vitrectomy, phacofragmentation and phacoemulsification in rabbit eyes.

\section{Methods}

Treatment followed the norms for the use of animals in research, established by the ethical code for animal experimentation of the Council for International Organization of Medical Science $(\mathrm{CIOMS})^{8}$ and the procedures were approved by the Ethics Committee for Animal Experimentation of the Ribeirao Preto
Medical School, University of Sao Paulo (068/2004).

We used 24 eyes of 12 male New Zealand albino rabbits weighing 2.0 to $2.5 \mathrm{~kg}$ obtained from the animal house of the Faculty of Medicine of Ribeirao Preto. The variation in IOP was determined during the surgical procedures of vitrectomy (eight eyes), phacofragmentation (eight eyes) and phacoemulsification (eight eyes).

Before each procedure, a preanesthetic intramuscular injection containing $2 \mathrm{ml}$ ketamine hydrochloride $(5 \mathrm{mg} / \mathrm{ml})$ and $1 \mathrm{ml}$ xylazine $(1 \mathrm{mg} / \mathrm{ml})$ was administered to each animal. Five to 10 minutes after the injection, with the animal sleeping, one ear was shaved and a marginal vein was punctured with a 19 gauge VeneScalp ${ }^{\circledR}$ coupled to a $20 \mathrm{ml}$ syringe containing $2.5 \%$ thiopentonarbital sodium 9 at the concentration of $10 \mathrm{mg} / \mathrm{ml}$. Anesthesia was maintained through this route by slow manual infusions of the drug determined by respiratory frequency and by the response to stimuli of each animal. Anesthesia was complemented with the instillation of $0.5 \%$ proximetacaine in the eye of the animal.

After anesthesia, one drop of $10 \%$ phenylephrine and one drop of $1 \%$ tropicamide were instilled in the eye to be operated in order to provoke mydriasis. Next, the surgical field was expanded by means of nasal and temporal canthotomy and opening of the conjunctiva at $360^{\circ}$ using a Daisy ${ }^{\circledR}$ instrument (Storz, St. Louis, MO, USA).

The variation in IOP in rabbits submitted to the procedures of vitrectomy via pars plana and facectomy with phacofragmentation or phacoemuslification was determined with a polyethylene cannula (PE50, Intramedic Inc.; Clay Adams, USA) linked to a computerized polygraph model MP 100 (Biopac Systems ${ }^{\circledR}$; Santa Barbara, CA, USA) through a transducer (Biopac Systems ${ }^{\circledR}$ ). After opening of the conjunctiva and sclerotomy (20 gauge) in the inferior nasal quadrant at $3 \mathrm{~mm}$ from the limbus, an extremity of the cannula was introduced into the vitreous chamber in the direction of the posterior pole and the other was connected to a transducer positioned at the same height in the eye of the animal. The variation in IOP provoked by the manipulation of the eye shifted the liquid column inside the cannula, generating a mechanical stimulus which, when reaching the transducer, was transformed into an electrical impulse. This impulse was then captured and filtered by the polygraph, which transferred it to the computer in the form of numbers and these, in turn, were converted to graphs by the Acqknowledge ${ }^{\circledR}$ software version 3.7.0 (Biopac Systems ${ }^{\circledR}$ ). The variation in pressure during the reading was $\pm 0.7 \mathrm{mmHg}$. Before each procedure the polygraph was recalibrated and the data recorded after the procedures were stored for later analysis.

In addition to IOP, the infusion pressure was also monitored during the surgeries. The infusion system was set up in a manner similar to that used for conventional surgery, with a cannula positioned inside the eye and connected to the serum catheter and to a suspended 
flask containing $500 \mathrm{ml}$ of balanced saline solution (Ophthalmos Ltda; Sao Paulo, Brasil). In all surgeries, the flask remained positioned at a fixed height of $40 \mathrm{~cm}$ above the eye level of the animal. For vitrectomy, the infusion was positioned in the vitreous chamber, while for phacofragmentation it was positioned inside the capsular sac. The infusion pressure was recorded by means of a transducer positioned between the infusion cannula and the serum catheter, maintained at the same height and at a distance of $20 \mathrm{~cm}$ from the eye of the animal. The infusion pressure during phacoemulsification, although recorded, was not considered since every time the apparatus closed the infusion system a pressure oscillation was produced that prevented an appropriate interpretation of the data.

The different aspiration pressures for each procedure were divided into weak, moderate and strong according to the indications of the equipment (Table 1). The parameter used during vitrectomy was 600 sections per minute. The parameters for phacofragmentation were: $74 \%$ power and $60 \%$ pulse and the parameters for phacoemulsification were $70 \%$ flow, $70 \%$ power, and $70 \%$ pulse. All procedures were performed by the same surgeon (EVS).

TABLE 1 - Division of the different aspiration pressures provided by the Daisy ${ }^{\circledR}$ apparatus (Storz, St. Louis, MO, USA) according to the type of intraocular surgery.

\begin{tabular}{lccc}
\hline \multirow{2}{*}{ Surgery } & \multicolumn{3}{c}{ Aspiration Pressure $(\mathrm{Mmhg})$} \\
\cline { 2 - 4 } & Weak & Moderate & Strong \\
\hline Vitrectomy & $0-40$ & $41-80$ & $81-120$ \\
Facofragmentation & $0-30$ & $31-60$ & $61-100$ \\
Facoemulsification & $0-30$ & $31-60$ & $61-100$ \\
\hline
\end{tabular}

\section{Results}

Figure 1 illustrates the variation in pressure recorded by the polygraph during vitrectomy. The mean IOP reading of all surgeries, together with the mean of the maximum and minimum values, is presented in Figure 2. The maximum peaks reached by vitrectomy, phacofragmentation and phacoemulsification were $38.4 \pm 5.2,33.7 \pm 3.4$ and $47.8 \pm 3.7 \mathrm{mmHg}$, respectively, during the procedure with strong aspiration. The minimum levels reached $0 \mathrm{mmHg}$ during vitrectomy and phacoemulsification, and $6.5 \mathrm{mmHg}$ during phacofragmentation in the step involving strong aspiration. Figure 3 presents the maximum and minimum peaks reached during the procedures according to the different aspiration pressures.

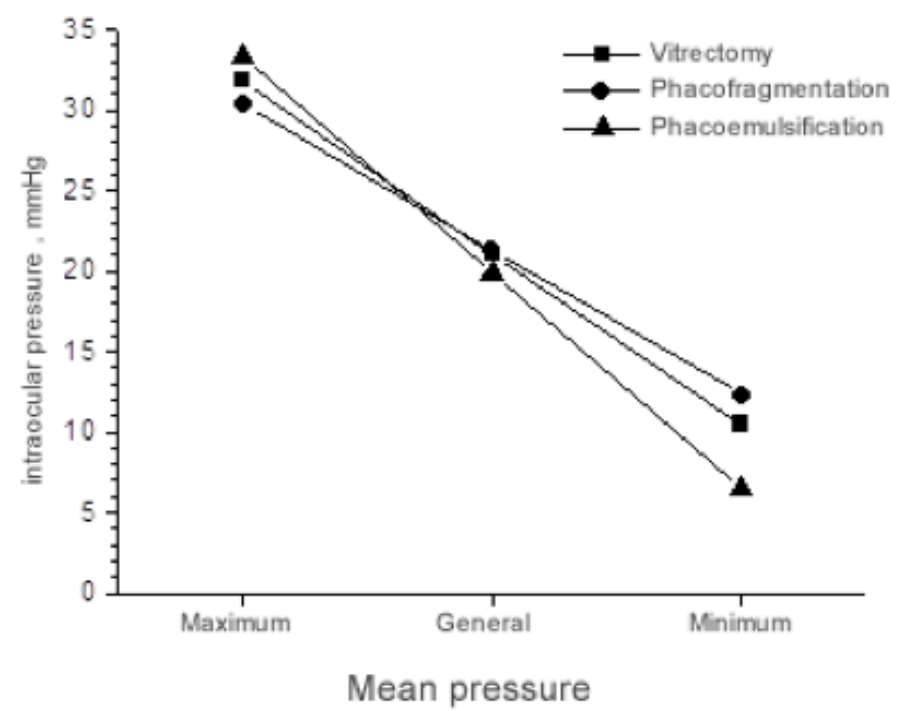

FIGURE 2 - Mean intraocular pressure recorded during procedures of vitrectomy, phacofragmentation and phacoemulsification in rabbit eyes; general mean and mean of maximum and minimum values.

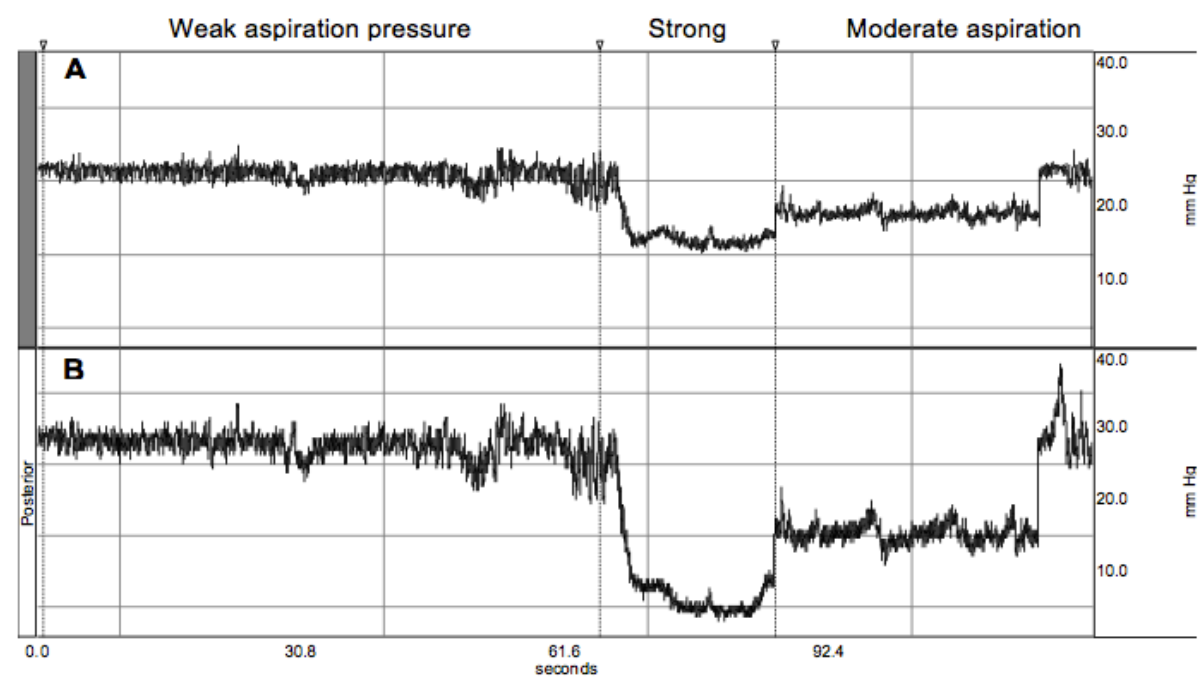

FIGURE 1 - Representative recording of infusion pressure (A) and intraocular pressure (B) fluctuation obtained with a polygraph (MP100, Biopac System ${ }^{\circledR}$ ) during vitrectomy in rabbit eyes, divided according to aspiration pressure. 


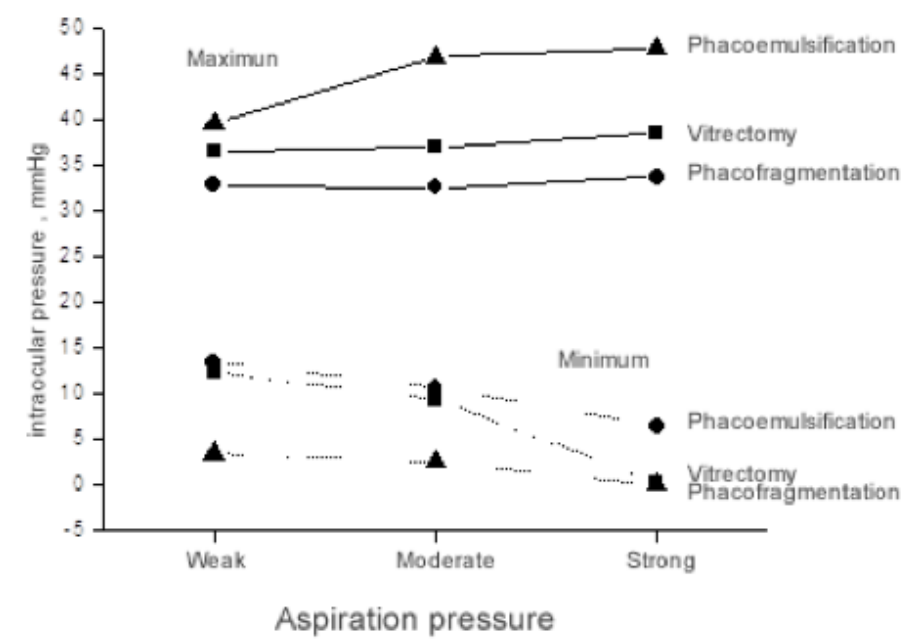

FIGURE 3 - Maximum and minimum values of intraocular pressure recorded during procedures of vitrectomy, phacofragmentation and phacoemulsification in rabbit eyes according to aspiration pressure (weak, moderate and strong).

Figure 4 presents the mean IOP for each procedure, comparing the various aspiration forces, i.e., weak, moderate and strong. Figure 5 (A and B) illustrates the general mean and maximum and minimum values of the different aspiration pressures.

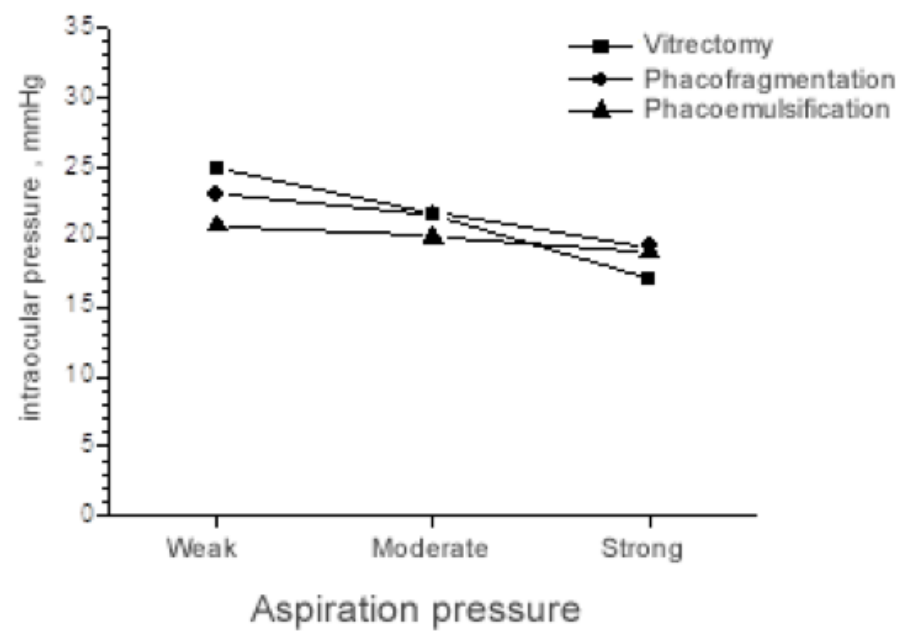

FIGURE 4 - Comparison of mean intraocular pressure recorded during procedures of vitrectomy, phacofragmentation and phacoemulsification in rabbit eyes according to aspiration pressure (weak, moderate and strong).
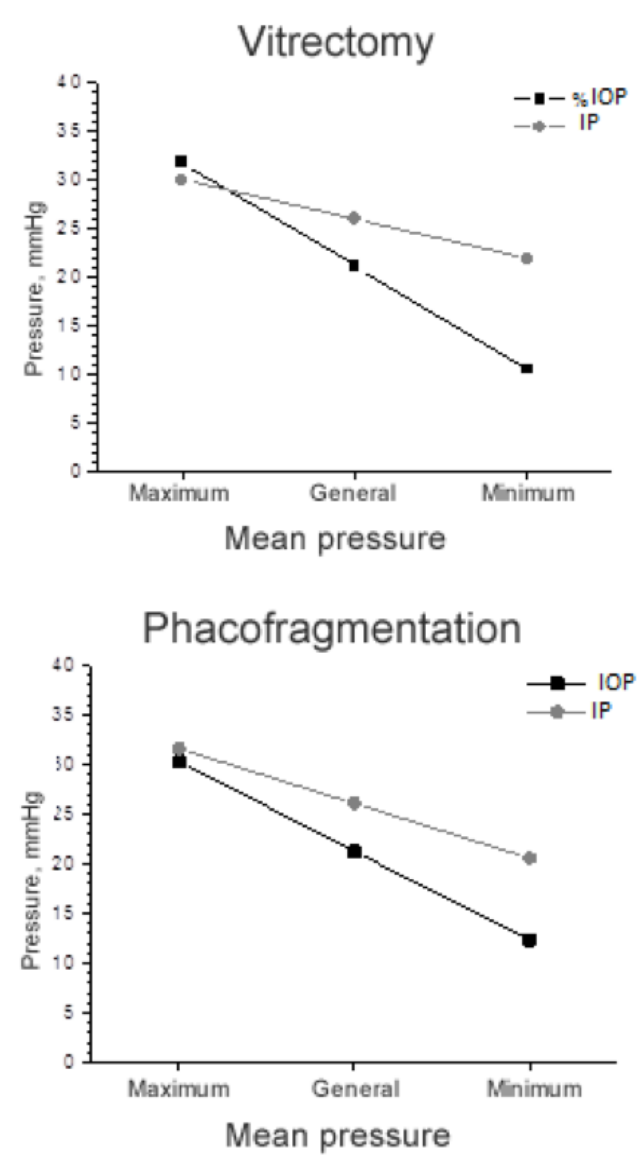

FIGURE 5 - Mean aspiration pressure with comparison of intraocular pressure and infusion pressure in vitrectomy and phacofragmentation according to the general mean pressure and maximum and minimum pressure in vitrectomy and phacofragmentation. $\mathrm{IOP}=$ intraocular pressure; $\mathrm{IP}=$ infusion pressure.

\section{Discussion}

The methods used in the present study were applied in a planned, rigid and pragmatic manner, with an attempt made to control the largest possible number of variables. However, it should be considered that the data presented here are intimately related to the animal model, the equipment, the material and the parameters employed, so that the results should be interpreted in the light of these limitations.

Rabbit eyes have been long used in experimental ophthalmologic surgeries ${ }^{9-11}$. In the present study, rabbits proved to be an excellent animal model both because of their availability and easy manipulation and because of their size and easy access to the eye. However, some aspects should be considered when this animal model is used for the study of variation in intraocular pressure. 
First, IOP is directly related to the thickness and rigidity of the sclera, which in rabbits is thinner and more elastic than in humans, representing a factor that may influence the final pressure ${ }^{12}$. It has been demonstrated that, when submitted to external forces of the same magnitude, pig eyes present more elevated pressure peaks than rabbits and that this is due to the greater scleral rigidity of pig eyes ${ }^{13}$. This fact permits the assumption that human eyes, under the same conditions, may show even higher pressure peaks than those detected in the present study. In addition to scleral rigidity, the crystalline can also influence the variation in pressure $^{13}$. We assume that, if the animals had presented cataracts, the result may have been different from that detected in the procedures of phacofragmentation and phacoemulsification since these eyes would have been submitted to different forces than those provoked by the present study. On the other hand, the lack of cataract induction was premeditated, since its presence would have caused an increase in the variables to be considered.

Other factors in addition to the animal model may have influenced the fluctuation of IOP during the procedures and therefore some measures were taken in order to minimize the effects of these influences. The instruments and equipment used were the same for all surgeries, as also were the parameters (height of the flask, pulse and potency of ultrasound, aspiration force, and sections per minute). The use of a polyethylene cannula (PE50) for the measurement of IOP proved to be important because this material does not undergo distension and because its large caliber prevents obstruction by vitreous humor. To confirm that the IOP reading was correct, during the pilot study a cannula was positioned in the anterior chamber and another in the vitreous chamber (data not shown). The result was a similar reading in the two chambers, so that the variations in pressure in the anterior chamber were transmitted to the vitreous chamber and vice versa. Previous studies have demonstrated that variations in pressure in the anterior chamber were transmitted simultaneously to the vitreous chamber at slightly lower intensities, which tended to become equal within a short time ${ }^{15}$. In addition, the presence of methylcellulose and air in the anterior chamber during facectomy may obstruct the cannula, thus compromising the reading of pressure. For these reasons, we opted for the use of only one cannula introduced into the vitreous chamber via the pars plana.

The present results showed that the variation in IOP during the proposed procedures was not wide, considering that simple manipulation of the eye, such as a massage, caused an increase in IOP to almost $100 \mathrm{mmHg}^{16}$, and that the greatest increase provoked by the experiment was close to $50 \mathrm{mmHg}$. Figure 3 illustrates the maximum and minimum values detected, with phacoemulsification showing greater variation, followed by vitrectomy and phacofragmentation.

The mean fluctuation of IOP, the mean of the maximum and the mean of the minimum was close to $20 \mathrm{mmHg}$ in all procedures and the maximum and minimum varied only 10 $\mathrm{mmHg}$ above or below this mean. The mean variation of IOP according to the pressure of aspiration showed that vitrectomy was the procedure that caused the greatest difference between weak and strong aspiration. This is believed to have occurred because this procedure uses infusion of large caliber, a fact that did not occur in the other procedures, thus justifying the higher IOP values with weak aspiration. In contrast, the lower mean value of the minimum IOP was justified by the fact that vitrectomy involved the highest aspiration pressure $(120 \mathrm{mmHg})$ compared to the other procedures $(100 \mathrm{mmHg})$.

Regarding the infusion pressure, the present study demonstrated that there is no good correlation between the pressure measured in the infusion system and the IOP (Figure 5). Figure 1 demonstrates the difference between IOP and perfusion pressure and the fact that wide fluctuations in IOP have repercussions on the infusion pressure as similar variations but of smaller magnitude. However, the pressure in the vitreous chamber should be the same as that of the infusion line under static conditions (with no fluid entering or exiting the eye) since both are part of a closed system in which pressure is equal at all points in the system ${ }^{16}$. However, under dynamic conditions (with liquid entering and exiting the eye, which occurs during vitrectomy and phacoemulsification, IOP differs from the infusion pressure ${ }^{14}$. In addition to confirming this finding, we observed that the behavior of these two pressures has a linear aspect. On this basis, we may state that, under the proposed conditions, IOP values below $30 \mathrm{mmHg}$ are accompanied by proportionally higher infusion pressures and, the lower the pressures, the greater the difference, as demonstrated in Figure 5. By extrapolating this reasoning, we may assume that, for IOP values above $30 \mathrm{mmHg}$, the infusion pressures are lower than IOP values, with this difference becoming more marked as we move away from this value. Thus, these results demonstrate the limitations of infusion pressure monitoring in the control of IOP.

\section{Conclusions}

Intraocular pressure (IOP) monitoring in rabbit eyes submitted to closed system intraocular procedures demonstrated that these procedures do not cause great pressure fluctuations, with greater IOP variation the higher the aspiration pressure. 
The infusion pressure did not show a good correlation with IOP since it presented greater stability and lower susceptibility to pressure oscillations.

\section{References}

1. Vasavada AR, Vasavada V, Vasavada VA, Praveen MR, Johar SR, Gajjar D, Arora AI. Comparison of the effect of torsional and microburst longitudinal ultrasound on clear corneal incisions during phacoemulsification. J Cataract Refract Surg. 2012 May;38(5):8339. doi: 10.1016/j.jcrs.2011.11.050.

2. Chan A, Leng T, Moshfeghi DM. Cannula-based 25-gauge vitreous tap and injection: a new surgical technique. Retina. 2012 May;32(5):1021-2. doi: 10.1097/IAE.0b013e318248e6ba.

3. Lee JY, Kim KH, Shin KH, Han DH, Lee DY, Nam DH. Comparison of intraoperative complications of phacoemulsification between sequential and combined procedures of pars plana vitrectomy and cataract surgery. Retina. 2012 Nov-Dec;32(10):2026-33. doi: 10.1097/IAE.0b013e3182561 fab.

4. Aizawa N, Kunikata H, Abe T, Nakazawa T. Efficacy of combined 25 -gauge microincision vitrectomy, intraocular lens implantation, and posterior capsulotomy. J Cataract Refract Surg. 2012 Sep;38(9):1602-7. doi: 10.1016/j.jcrs.2012.05.028.

5. Hughes WF. Quantification of ischemic damage in the rat retina. Exp Eye Res. 1991 Nov;53(5):573-82. PMID: 1743256.

6. Resta V, Novelli E, Vozzi G, Scarpa C, Caleo M, Ahluwalia A, Solini A, Santini E, Parisi V, Di Virgilio F, Galli-Resta L. Acute retinal ganglion cell injury caused by intraocular pressure spikes is mediated by endogenous extracellular ATP. Eur J Neurosci. 2007 May;25(9):2741-54. PMID: 17459106.

7. Tamai K, Toumoto E, Majima A. Protective effects of local hypotermia in vitrectomy under fluctuating intraocular pressure. Exp Eye Res. 1997 Dec;65(6):733-8. PMID: 9441696.

8. Howard-Jones N. A CIOMS ethical code for animal experimentation. WHO Chronicle. 1985;39(2):51-6. PMID: 4090462.

9. Eifrig DE, Doughman DJ. Intraocular lens in laboratory animals. Ophthalmic Surg. 1977 Jun;8(3):149-52. PMID: 904867.

10. Polack FM, Sugar A. The phacoemulsification procedure. II. Corneal endothelial changes. Invest Ophthalmol. 1976 Jun;15(6):458-69. PMID: 931690.

11. Honda Y, Negi A, Kawano S. Ion movement in and out of the vitreous space after vitrectomy. An experimental study on the in vivo eyecup preparation of rabbits. Invest Ophthalmol Vis Sci. 1981 Jul;21(1 Pt 1):126-30. PMID: 7251296.

12. Barbazetto IA, Liang J, Chang S, Zheng L, Spector A, Dillon JP. Oxygen tension in the rabbit lens and vitreous before and after vitrectomy. Exp Eye Res. 2004 May;78(5):917-24. PMID: 15051473 .
13. Lauretti Filho A, Romão E. Ocular rigidity: experimental and clinical study. Rev Bras Oftalmol. 1970 Jun;29(2):115-71. PMID: 5520777.

14. Moorhead LC, Armeniades CD. Variations in intraocular pressure during closed-system surgical procedures. Arch Ophthalmol. 1986 Feb;104(2):269-72. PMID: 3947302.

15. Bui BV, Edmunds B, Cioffi GA, Fortune B. The gradient of retinal function changes during acute intraocular pressure elevation. Invest Ophthalmol Vis Sci. 2005 Jan;46(1):202-13. PMID: 15623775.

16. Honda Y, Kawano S, Negi A, Koizumi K. Pressure profile of ophthalmic surgical procedures: an experimental study on the rabbit eye. Ophthalmic Surg. 1982 May;13(5):387-91. PMID: 7099528.

\section{Acknowledgement}

To Paulo Roberto Barbosa Évora, PhD, for technical support.

\section{Correspondence:}

Profa. Dra. Maria de Lourdes Veronese Rodrigues

Departamento de Oftalmologia, Otorrinolaringologia e Cirurgia de Cabeça e Pescoço

Faculdade de Medicina de Ribeirao Preto - USP

14048-900 Ribeirão Preto - SP Brasil

Tel.: (55 16)3602-2862

mdlvrodr@fmrp.usp.br

Received: June 23, 2014

Review: Aug 25, 2014

Accepted: Sep 22, 2014

Conflict of interest: none

Financial sources: Coordination of Improvement of Higher Academic Staff (CAPES) and Foundation for Education, Research and Assistance Support (FAEPA), Medical School of Ribeirao Preto, University Hospital.

${ }^{1}$ Research performed at Cardiovascular and Endotelial Physiology Laboratory, Surgery and Anatomy Department, and Ophthalmology, Otorhinolaringology and Head and Neck Surgery Department, Medical School of Ribeirao Preto, University of Sao Paulo (FMRP-USP), Brazil. Part of $\mathrm{PhD}$ degree thesis, Postgraduate Program in Ophthalmology, Otorhinolaringology and Head and Neck Surgery, FMRP-USP. Tutor: Profa. Dra. Maria de Lourdes Veronese Rodrigues. 